\title{
Comparison of Shear Bond Strength of Edgewise Bracket Bonded to Composite Restoration by Using Three Regimes of Orthodontic Adhesive Systems (In Vitro Study)
} Hasan Sabah Hasan*

B.D.S., M.Sc.; Assist. Lect., Department of Orthodontics, Khanzad specialist and teaching centre, General health directorate in hawler, Ministry of health KRG.

\section{Bayan Abdulla Hassan}

B.D.S., M.Sc., Ph.D.; Assist. Prof., Department of Orthodontics, College of Dentistry, Hawler Medical University.

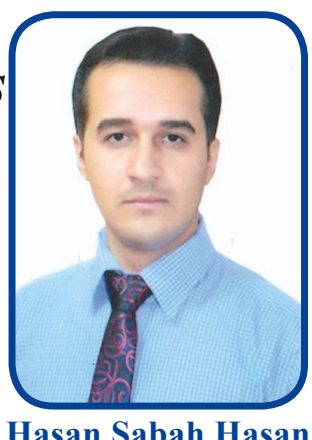

\begin{abstract}
Introduction: Direct bonding of orthodontic attachment has removed some of the esthetic concerns many adults previously had when considering orthodontic therapy. With an increase in adult treatment comes the challenge of direct bonding to non-enamel surface, such as composite restoration. This in vitro study was designed to compare the effect of using three regimes of orthodontic adhesion systems on shear bond strength when bonded edgewise brackets to composite restoration.

Materias and methods : The study samples were randomly divided into three groups (30 specimens each).Group I using resilience orthodontic adhesive material (4thgeneration) consist from acid-etching, primer and adhesive; group II using heliosit orthodontic adhesive (1stgeneration) consist from acid-etching and adhesive without bonding agent; group III using self-etching/self-bonding orthodontic adhesive (Totalcem) (7thgeneration).

Results: The result of the study showed that the light cured bonding adhesive resilience orthodontic (group I) has the highest mean of shear bond strength (33.7 Mpa) followed by dual-cure automix bonding self-etch/self-bonding adhesive resin cement (23.6 Mpa). While the light cured bonding adhesive heliosit showed the lowest mean of shear bond strength (18.04 Mpa). The cohesive failure (score 2) was the predominant mode of the bond failure in group (I) (4thgeneration) of this study, also the adhesive-composite interface failure was the predominant especially in group (II) (1stgeneration). In group (III) (7thgeneration) while the adhesive -composite interface failure was predominant, but cohesive failure (score 2) and composite detachment (score 4) was found but in less percentage if compared with adhesive-composite interface failure in the same group.
\end{abstract}

KEYWORDS

Orthodontic adhesive material, Universal testing machine, Edgewise brackets

CITE THIS ARTCLE:

Hasan H, Hassan B. Comparison of shear bond strength of edgewise bracket bonded to composite restoration by using three regimes of orthodontic adhesive systems (in vitro study). Iraqi Dental Journal 2015; 37(1):32-31. http://www.iraqidentaljournal.com

$$
\begin{aligned}
& \text { مقارنة بين قوة رباط القص للحاصر الجانبي المرتبط بحشوة الراتنج المركب بإستخدام ثلاثة أنظمة من نُظم لاصقة } \\
& \text { لتقويم الأسنان } \\
& \text { حسن صباح حسن } \\
& \text { مدرس مساعد - مرس صباح حسن خسن اد التعليمي } \\
& \text { استاذ مساعد ـ قسم التقويم - كلية طبد الله حسن الاسنان , جامعة هنة لير الطبية }
\end{aligned}
$$

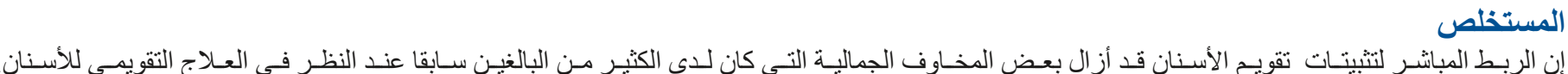

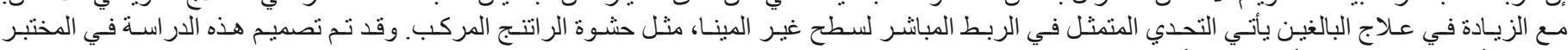

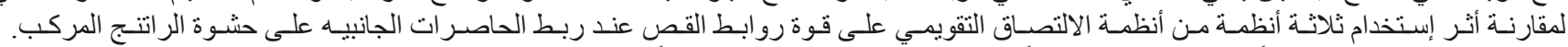

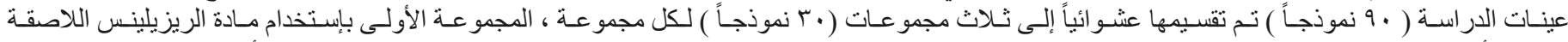

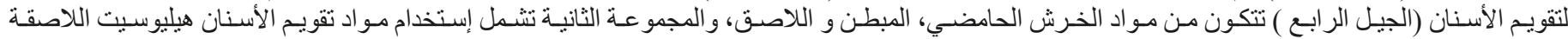

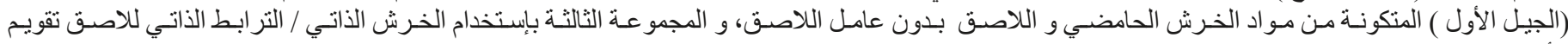

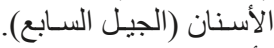

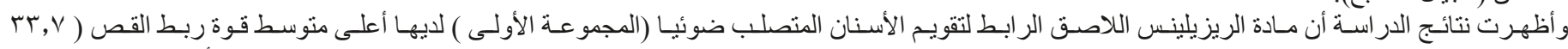

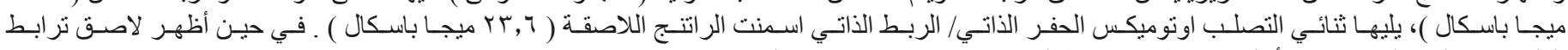

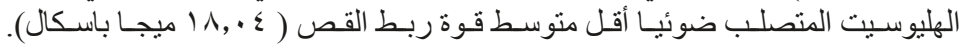

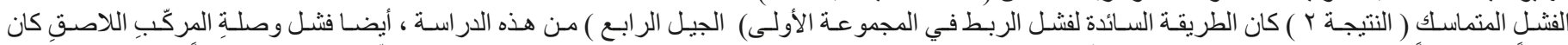

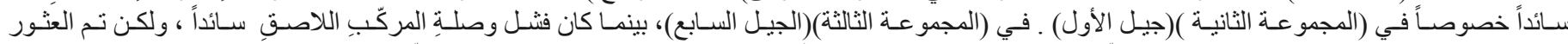

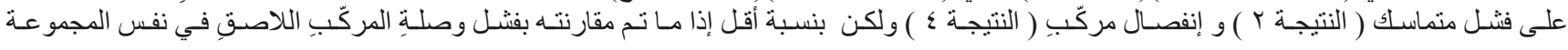

\section{INTRODUCTION}

Orthodontists recognize the ability to bond brackets successfully to natural tooth structure. With

the composite resin type of restorative material, the orthodontist will need to bond composite adhesive 
directly to composite material(Bright\&Shannon,1980; Al-Bers,2005; Albaladejo et al.,2011). Excessively high bond strength values are undesirable because of the increase the debonding forces needed, resulting in possible damage to composite restoration (Duggal, 2011). The big problem that faces orthodontist is debonding of the brackets specially in cases when it fixed on restoration like composite. Therefore a roughened surface will need in order to bond the adhesive composite material to the substrate composite restoration, so this roughness will lead to distraction of the composite restoration surface and weaken it (Nilsoon \&Alaeddin, 2000). So to prevent this from occurrence and with the development of newly adhesive bonding material, so the aim of this study was to compare shear bond strength of three different regimes of adhesive bonding material for bonding the orthodontic edgewise stainless steel brackets on composite restoration without doing any scratching on its surface and to estimate the mode of adhesive failure.

\section{MATERIAS AND METHODS}

\section{Construction of composite restoration sample:}

Ninety clear central incisor celluloid crowns were used to construct ninety light cure prime-dent composite restoration (A2 shade) as central incisor like shape. The composite was loaded inside the celluloid crown of central incisor in three layers of $3 \mathrm{~mm}$ in depth by using plastic condenser instrument(as enough thickness for making good curing by the visible LED light cure unit with intensity power of $1200 \mathrm{~mW} / \mathrm{cm}^{2}$ and wavelength 480 um (Discuss Ivoclar Vivident) (Cavalcanti et al.,2004), the first layer was applied and condensed very well to remove all the air bubble by using plastic condenser in the celluloid crown then it was cured by using a visible LED light cure unit from labial surface of the crown for 30 second and from the lingual surface also for 30 seconds (Al-Hashimi, 2001). After curing of the first layer the second layer was applied inside the crown also for about $3 \mathrm{~mm}$ as in first layer, but before curing process a screw post was inserted inside the composite restoration, then good condensation and adaptation of the composite restoration around the screw was made by using plastic condenser in order to make good retention and adaptation also to prevent air bubble formation between the screw and composite restoration as recommended by Chay et al.(2007) as shown in figure 1. The restoration was cured as mention in the first layer. The final third layer was loaded around the screw also for $3 \mathrm{~mm}$ as in second layer and was cured from the labial and lingual aspect of the celluloid crown for 30 seconds for each side.
The screw was useful in the retention of the composite restoration to the acrylic block that was constructed after the composite restoration sample finished and also helped in the surveying of the middle third of the labial surface of the composite restoration. Then the celluloid crown was removed and ended with a composite restoration as central incisor with screw at the cervical end of restoration.

\section{Surveying of the composite sample:}

Aglass slide was placed on a table. The restoration sample was fixed on the glass slide in a vertical position using sticky wax. The glass slide with the fixed restoration sample was placed at the surveyor table (the table of surveyor device at zero angle), then the middle third of the buccal surface of the composite restoration was surveyed in order to orient it parallel to the analyzing rod of the surveyor and touch it. So that the force from the chisel-edge rod of universal test machine will be applied at a right angle to the composite-bracket interface as recommended by AlKhateeb (2012).

\section{Construction of the acrylic block:}

After surveying had been done, two metallic L-shape like box were positioned around the fixed composite restoration in such way that the crown was protruded from the metallic L-shape box, then each end of this metallic L-shape was fixed with sticky wax in order to prevent any movement or dislodgement. Cold-cure acrylic was mixed and poured around the restoration to the level that the acrylic will touch the composite at cervical line. After setting of the acrylic resin had been complete the two L-shape metal box were opened and the acrylic block was finished and polished as recommended by Al-Khafaji, (2000)as shown in figure 2.

\section{Sample hydration:}

All samples were hydrated in deionized distilled water at $37^{\circ} \mathrm{C}$ in incubator for one week before bonding procedure of the edgewise brackets and for 24 hour after bonding of the brackets in order to allow adequate water sorption of the composite restoration to simulate the effect of the oral environment on the composite restoration depending on Woolaver (2000), Al- Dabbagh (2008) and Al-Shamaa (2009).

\section{Sample preparation before bonding process:}

The labial surface of all composite restorations was polished using non-fluoridated pumice with rubber cup. Each composite restoration was washed with water spray for 10 seconds, the surface was dried for 10 seconds (Ajlouni et al.,2005; Al-Shamaa, 2009; Garma et al.,2011). The dryness of the labial surface of the composite restoration had been done by air spray pumping device that had been constructed especially for this study (the device consist from 
electrical air pump with specially changing in its inside coil in order to increase its air pumping ability, also have some modification in its tube in order to simulate the air spray (triple syringe) of the dental chair, this device had ability to give a full surface dryness without any water drop, small portable size, easy to use, low coasty, have effected result, easy to replace the spray tube or use disposable one and had no noisy sound).

\section{Sample grouping:}

The samples (90 composite restorations) were randomly divided in to three groups according to the three types of orthodontic adhesive material that were used for bonding the stainless steel edgewise brackets to the composite restoration sample as follow: Group I consist from 30 samples, $4^{\text {th }}$ generation of resilience orthodontic adhesive (acidetching+primer+adhesive). Group II consist from 30 samples, $1^{\text {st }}$ generation heliosit orthodontic adhesive (acid-eching+adhesive). Group III consist from 30 samples, $7^{\text {th }}$ generation totalcem self-eching/selfadhesive resin.

\section{Bonding procedure:}

The bonding procedure was different according to each type of sample group, in the first group of study the light cure bracket bonding adhesive Resilience orthodontic was used, $37 \%$ phosphoric acid gel was applied on the middle third of the labial surface of the composite restoration for 30 seconds, rinsed with water spray for 20 seconds, dried with air spray drying device for 10 seconds according to Bishara et al.(2005). Then a thin coat of Resilience orthodontic primer was applied on the etched surface of the composite restoration by using dental brush, curing it for 10 seconds, the Resilience orthodontic adhesive was applied on the base of the edgewise bracket, positioning it on the middle third of the labial surface of the composite restoration vertically to the long axis of it by using a bracket holder and bracket positioner (distance about $4.5 \mathrm{~mm}$ from the incisal edge as recommended by Bishara et al.(2005). A constant load was applied on the bracket for 10 seconds by fixing a $250 \mathrm{gm}$ load on the upper part of the vertical arm of the surveyor and fixing a hard rubber polishing bur in the lower part of the vertical arm of the surveyor and putting it in contact with bonded bracket to ensure that each bracket will seat under an equal force also to ensure a uniform thickness of the adhesive and to prevent air entrapment which may affect bond strength as described by Nemeth et al.(2006)and AlShamaa (2009). Any access of adhesive material was carefully removed by sharp prop (Al-Khafaji, 2000) as shown in figure 6 . The adhesive was cured by using a LED visible light cure unit for 40 second.

In the second group, after etching process had been completed as in the first group. The heliosit orthodontic adhesive ( $1^{\text {st }}$ generation) was applied on the base of the edgewise bracket after holding it with bracket holder, positioning it on the composite restoration and the bonding procedure was completed as in first group. In the third group, the Dual-cure Automix Bracket bonding self-etching/self-Adhesive Resin $\left(7^{\text {th }}\right.$ generation) was used. So the phosphoric acid gel was not use. the self-etching/self-Adhesive resin was applied on the base of edgewise bracket and the bonding procedure was completed as in first group, Following the manufacturer's instructions it was left for $1.5 \mathrm{~min}$ to let the effect of its etching and curing process completed with visible LED light cure unit for $1 \mathrm{~min}$. and 20 seconds, after that each sample was left for $1 \mathrm{~min}$ to complete the dual curing effect (Fig. 3).

\section{Shear bond strength test:}

The shear test was carried using a Universal testing machine (Gunt, Hamborg, Germany) with speed $(5 \mathrm{~mm} / \mathrm{min})$ as shown in figure 4 . Each specimen was fixed in the lower jaw of the universal testing machine, so that the base of the bonded brackets was parallel to the shear force direction and inciso-gingival load was applied to the restoration-bracket interface from knife edge rod until debonding occurred, then the maximum load necessary to debonded the brackets was recorded (Uysal \& Sisman, 2008). Each debonded brackets was kept in labeled container indicating group with its corresponding composite restoration to estimate the adhesive remnant index (ARI) according to Alkhateeb (2012).

\section{Estimation of the Adhesive Remnant Index (ARI):}

The debonded bracket and the composite restoration surface of each sample was inspected using a stereomicroscope at magnifying power $40 \mathrm{x}$ (Motic,USA) to determine the predominant site of failure similar to method mention by Bishara et al. (2004)and Polat et al.(2004). The site of bond failure was scored according to Wang et al. (1997), as follow:

- Score 1: Between the bracket base and adhesive.

- Score 2: Cohesive failure within the adhesive itself, with some of the adhesive remained on the bracket base and some remained on the composite restoration surface.

- Score 3: Between adhesive and composite restoration surface.

- Score 4: composite restoration detachment (Fig.5).

\section{Statistical analysis:}

The statistical package for social science (SPSS) version 19 (2012) was used for data entry and analysis. While Microsoft Excel 2007 was used for plotting the groups.

P level of 0.05 was accepted as statistically significant at the following levels: 


\section{Orthodontics}

- $\mathrm{p}>0.05$ Non significant

- $0.01<\mathrm{p}<0.05$ Significant

- $\mathrm{p}<0.01$ Highly significant

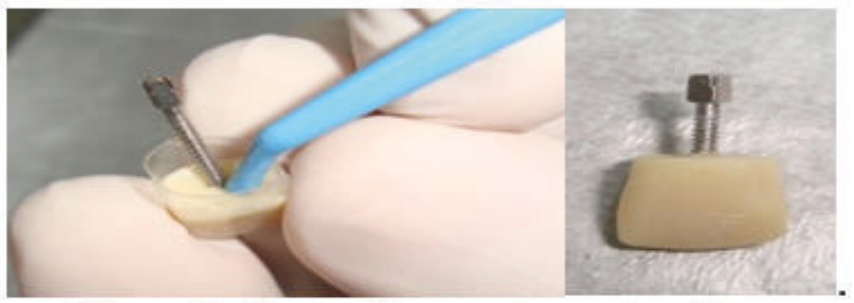

Figure 1: Composite sample construction.

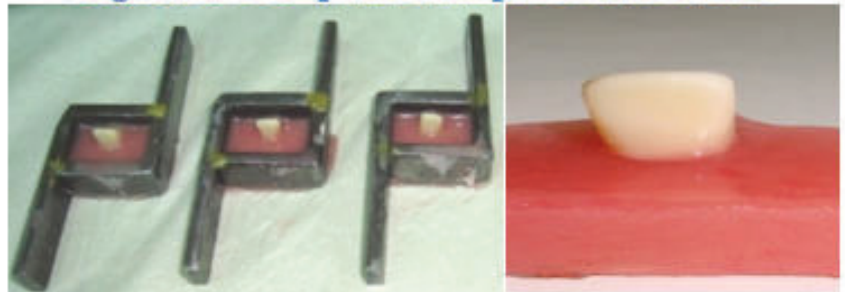

Figure 2: Acrylic block construction.

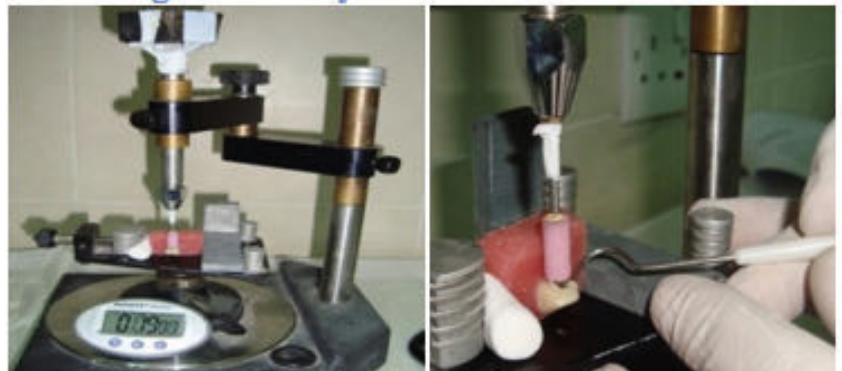

Figure 3: Bracket fixation process.

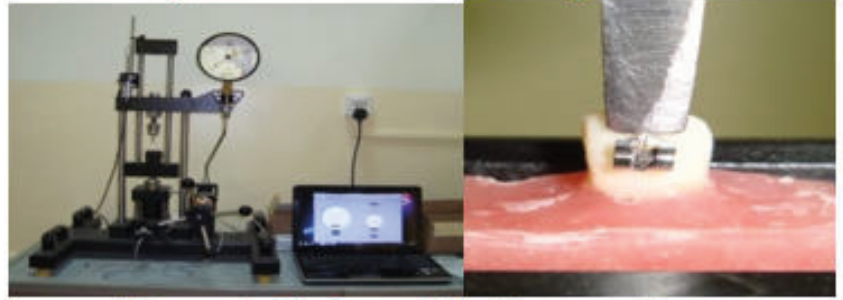

Figure 4: Universal testing mashine.

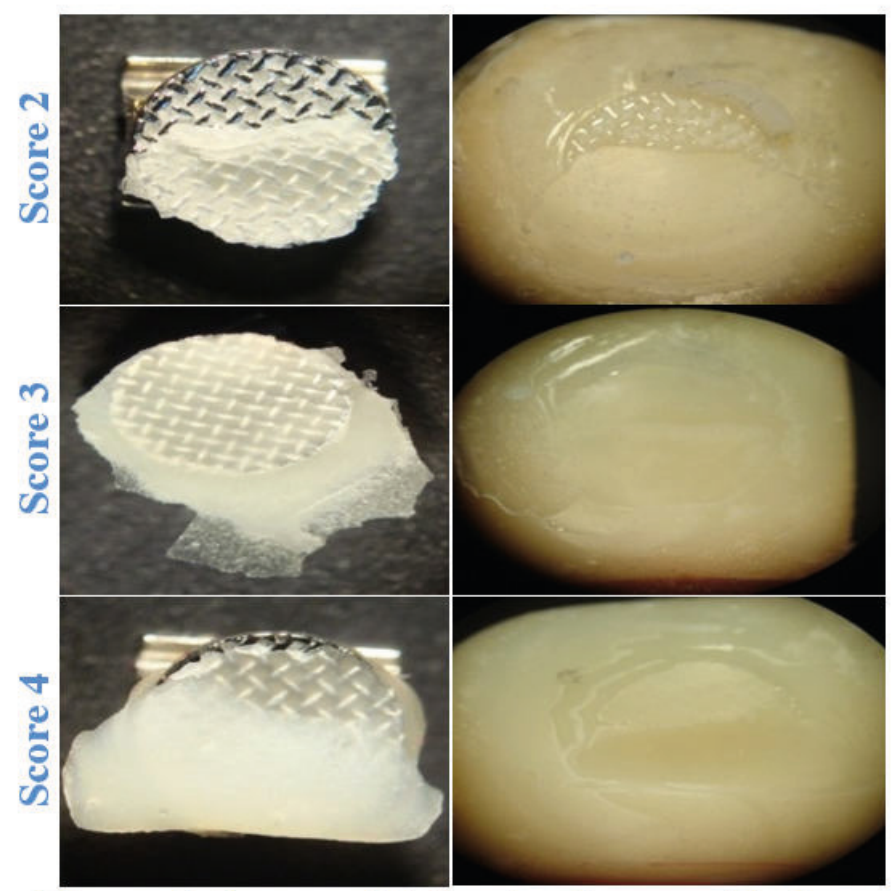

Figure 5: Scores of Adhesive Remnant Index.

\section{RESULTS}

\section{Shear Bond Testing Values:}

The descriptive statistics (mean, standard deviation with minimum and maximum values and median) of the shear bond strength of each group are presented in table 1.

From this descriptive statistics, it is clearly obvious that group I (light cured bracket bonding adhesive Resilience orthodontics) have the highest mean of shear bond strength (33.704.98 \pm ) of all groups, followed by group III (Dual- cure Automix Bracket bonding self-etching/self-Adhesive Resin Cement) with mean of shear bond strength (23.616.43 \pm ) then group II (Light cured Brackets bonding Adhesive Heliosit orthodontic) have mean of shear bond strength (18.043.58 \pm ). The statistical analysis of data by one way analysis of variance (ANOVA) showed a statistically highly significant difference $(p<0.001)$ in shear bond strength of all the groups as presented in table 2 .

\section{Least Significant Difference Test:}

LSD-test was used to investigate where the significant difference did occur. The PostHoc test (LSD) showed significant results between all the groups. So the result showed that there were statistical significant difference between:

- Group (I) vs. group (II).

- Group (I) vs. group (III).

- Group (III) vs. group (II )

\section{Mode of bond failure:}

The sites of bond failure of the specimens are presented in table 3. The failure site for group I occurred as cohesive failure (score 2) in percentage $76.7 \%$ (23 samples) and occurred at adhesivecomposite interface (score 3$)$ in percentage 20\% (6 samples) and occurred as composite detachment (score 4) in 3.3\% (1 sample). The failure side for group II occurred as failure in adhesive-composite surface interface in percentage 96.7\% (29 samples) and occurred as cohesive failure in percentage 3.3\% (1 sample). The failure site of group III occurred in the adhesive-composite interface in percentage 53.3\% (16 samples) and as cohesive failure in percentage $26.7 \%$ (8 samples) and as composite detachment in percentage $20 \%$ (6 samples).

The statistically chi-square test showed a highly significant difference between the groups in the site of bond failure $(\mathrm{p}<0.001)$ as showed in table 3 . The bond strength of orthodontic brackets on tooth surface is of great concern to orthodontists. The bonding of orthodontic brackets to enamel has been well-documented in orthodontic literature (Bishara 


\section{Orthodontics}

et al.,2005). With the demand for adult orthodontic treatment, clinicians need to acquire more knowledge about bonding to non-enamel surface. In clinical use, the bond must be strong enough to withstand orthodontic and chewing forces (Duggal, 2011).

Table 1: Descriptive statistics of shear bond strength (Mpa) of all groups.

\begin{tabular}{|c|c|c|c|c|c|c|}
\hline Group & N & Min. & Max. & Mean & SD. & Median \\
\hline Group I & 30 & 23.88 & 39.80 & 33.70 & 4.98 & 33.70 \\
\hline Group II & 30 & 15.92 & 23.88 & 18.04 & 3.58 & 18.04 \\
\hline Group III & 30 & 15.92 & 31.84 & 23.61 & 6.43 & 23.61 \\
\hline
\end{tabular}

Table 2: Comparison of means of shear bond strength of the three study groups (by ANOVA- test).

\begin{tabular}{|c|c|c|c|c|}
\hline Groups & $\boldsymbol{N}$ & Mean & SD & p value \\
\hline Group I & 30 & 33.697 & 4.983 & $<0.001^{*}$ \\
\hline Group II & 30 & 18.043 & 3.580 & \\
\hline Group III & 30 & 23.615 & 6.437 & \\
\hline Total & 90 & 25.118 & 8.259 & \\
\hline
\end{tabular}

*PostHoc test (LSD) showed significant results between all the groups

Table 3: Failure site in shear test of all groups.

\begin{tabular}{|l|c|c|c|c|} 
& Group I & Group II & Group III & \multirow{2}{*}{ p *value } \\
\cline { 2 - 5 } & No. (\%) & No. (\%) & No. (\%) & \\
\hline Score 2 & $23(76.7)$ & $1(3.3)$ & $8(26.7)$ & $<0.001$ \\
\hline Score 3 & $6(20)$ & $29(96.7)$ & $16(53.3)$ & \\
\hline Score 4 & $1(3.3)$ & $0(0)$ & $6(20)$ & \\
\hline Total & $30(100)$ & $30(100)$ & $30(100)$ & \\
\hline
\end{tabular}

* p calculated by Fisher's exact test

\section{DISSCUSION}

The direct bonding of metal bracket to PrimeDent composite restorations by using different regimes of orthodontic adhesive resin, demonstrated bond strength that was clinically accepted (15.9-39.8 Mpa). This could be attributed to its content of bifunctional acrylates, which cross-link to provide increase mechanical strength and resistance to weakening in the presence of water (Haselton et al.,2002). In groupI were etched with $37 \%$ phosphoric acid then coated with bonding agent, it was demonstrate a high mean of shear bond strength (33.7) and showed a highly statistically significant difference between this group and other groups (Group II and III). This result could be attributed to the effect of the polishing of the adhered surface of the composite restoration with pumice that leaded to remove the contamination and expose the fresh swollen restoration lead to formation of slightly retentive surface, this freshly restoration has a lot of unreacted methylate groups with coupling saline of the composite restoration, also with the effect of the acid to produces micro-retentive surface that dissolve the glass particles of the filling leaving gaps or porous that allow micromechanical retention by bonding agent as recommended by Yap et al.(2000) and Rathke et al.(2009). Appling bonding agent based on chloro-phosphate esters of Bis-GMA resin and polar nature of the phosphate group may contribute to bond with the inorganic filler component of the composite, also the low viscosity of this bonding agent produces a small contact angle and good wetting properties and high penetration coefficient (Azarbal et al.,2009). The penetration coefficient of the bonding agent evidently wets the exposed filler particles, prevent formation of air voids and improves adaptation between separate layer, so the present of the micro-mechnical interlocking by the effect of etching and polishing procedure with the effect of the bonding agent all of this may contributed in high mean of shear strength in this group of this study (Azarbal et al.,2009). In groupII were etched with $37 \%$ phosphoric acid then the adhesive was directly applied without coated with any primer, it was demonstrate a low mean of shear bond strength (18.04) in comparing with other groups (Group I and III). With the effect of polishing and acid-etching. This result could be attributed the absent of the bonding agent between adhesive and composite restoration surface, so the free radical polymerization reaction in a covalent chemical bond between the monomers of the newly applied polymerizing material (adhesive) with the unreacted remaining monomers on the surface of the previously polymerized substratum (composite restoration), this covalent chemical bond is not strong as in compare with that occur in present of bonding agent between the adhesive and composite restoration (Li, 1997 ; Craig \& Ward, 1997). In groupIII was demonstrate a high mean of shear bond strength (23.6) in comparing with mean of group II and showed a statistically difference with it, but showed a low mean in comparing with that of group I.This result could be attributed to the etching performance of self-etching primer is weaker than that of $37 \%$ phosphoric acid etching. As a result, the self-etching primer shows a more conservative etch pattern but has fewer adhesive penetrations, leading to lower bond strength in comparing to the mean of shear strength of group I (Scougall Vilchis et al.,2007). A self-etching primer consists of acidic adhesive monomer, deionized water, activator, and stabilizer. The bonding performance of an adhesive monomer can be mainly influenced by its hydrophilic acid moieties (Velo et al., 2002). 


\section{Failure sites:}

Concerning the adhesive remnant index scores which give the indication about the type of bond failure for each group, so score II was most predominant $(76.7 \%)$ in the samples bonded with resilience orthodontics adhesive (Group I) using edgewise brackets, also it was predominant $(26.7 \%)$ in the samples bonded with self-etching/selfbonding adhesive cement (Group III), and it was less commend $(3.3 \%)$ in the samples bonded with Heliosat orthodontic adhesive (Group II). This could be attributed to the Self-Curing type that has a dual cure process; acid base reaction and resin monomers polymerization, providing stronger chemical bonding within the adhesive itself cause increase the stiffness and physical properties of the adhesive (Fricker, 1998). Also it could be encounter to the bonding of orthodontic adhesive to polycarbonate attachment which is typically by mechanical and is achieved by creating microretentive elements in the base (Brantly \& Eliades, 2001). So, a good mechanical interlock could be obtained between the adhesive and brackets base, so that the retention of the adhesive to the attachment base is greater than that within the adhesive itself. Harari et al.(2003) and Sarac et al.(2007) reported that cohesive failure (score 2 of ARI) is preferred to avoid composite restoration fracture during debonding which clinically indicates the long-term integrity of the composite restoration. Score3 indicate failure at adhesive-composite interface, since the bond failure occurs usually at the area of least resistance which mean that the bond strength between the adhesive-bracket interface and cohesive bond strength of the adhesive itself were stronger than the bond strength between the adhesive and composite restoration. It was more predominant $(96.7 \%)$ in samples bonded with heliosit orthodontic adhesive (Group II) using edgewise brackets, also was predominant $(53.3 \%)$ in samples bonded with selfetching/ self-bonding adhesive cement (GroupIII), and was less predominant (20\%) in samples bonded with resilience orthodontics adhesive (Group I). Score 4 the composite detachment was predominant (20\%) in samples bonded with self-etching/ self-bonding adhesive cement (Group III) using edgewise brackets, also it was less predominant (3.3\%) in samples bonded with resilience orthodontics adhesive (Group I), and it was $0 \%$ in sample bonded with Heliosit orthodontic adhesive (Group II). This could occur due to high bond strength at the composite- adhesive interface that results in composite detachment (Fricker, 1998).

\section{CONCLUSION:}

1.The use of the $4^{\text {th }}$ generation (Resilience orthodontic adhesive) is the most suitable material for bonding orthodontic brackets to composite restoration.

2.The use of $7^{\text {th }}$ generation self-etching/self-bonding orthodontic adhesive for bonding orthodontic brackets to the compositerestoration provide enough shear bond strength to withstand orthodontic forces recommended for orthodontic tooth movements.

3.The use of $1^{\text {st }}$ generation (Heliosit orthodontic adhesive) provide the lowest shear bond strength among the groups.

4.The cohesive failure was the predominant mode of the bond failure in groupI $\left(4^{\text {th }}\right.$ generation $)$ of this study, also the adhesive -composite interface failure was the predominant especially in group II( $\left({ }^{\text {st }}\right.$ generation $)$. In group III $\left(7^{\text {th }}\right.$ generation $)$ while the adhesive-composite interface failure was predominant, but cohesive failure (score 2) and composite detachment(score 4) was found but in less percentage if compared with adhesivecomposite interface failure in the same group.

\section{REFERENCES}

1. Bright DS, Shannon IL. Effect of phosphoric acid gel on tensile strength of direct bonded orthodontic brackets. Int $\mathbf{J}$ Orthod 1980; 18(1):7-13.

2. Al-Bers HF. Tooth-Colored restoration; principles and technique. $9^{\text {th }}$ ed. Hamilton, London, 2005.

3. Albaladejo A, Montero J, Gomez de-diego R, Lopez-valver de A. Effect of adhesive application prior to bracket bonded with flowable composite. Angle Orthod 2011; 81(4):71620.

4. Duggal R. Comparison of shear bond strength of new generation bonding adhesive with two other generation adhesive using metal bracket (In vitro study). JADA 2011; 5(3):351-8.

5. Nilsoon RV, Alaeddin S. Factor affecting the shear bond strength of bonded Composite inlays. Int J Prosthod 2000; 13(2):52-58.

6. Cavalcanti AN, Lavigne C, Fontes CM, Mathias P. Microleakage of the composite repair interface: effect of different adhesive system. J Appl Oral Sci 2004;12(3):21922.

7. Al-Hashimi AG. Comparison of the shear bond strength of immediate and delayed repair of the light cured composite restoration (In vitro study). Master thesis. Conservative Department, University of Bagdad, 2001.

8. Chay SH, Wong SL, Mohamed N, Chia A, Yap AUJ. Effect of the surface treatment and aging on the bond strength of orthodontic brackets to provisional material. Am J Orthop Dentaofac Orthop 2007; 132(5):577-85.

9. Al-khateeb HM. Shear bond strength of different lingual buttons bonded to wet and dry enamel surface with resin modified glass-ionomer cement (In vitro comparative study). Master thesis, Orthodontic department, University of Baghdad, 2012.

10. Al-khafaji HF. Shear and tensile bond strength of 


\section{Orthodontics}

orthodontic adhesive with stainless steel brackets (In vitro study). Master thesis. Orthodontic department, University of Baghdad, 2000.

11. Woolaver J. The Shear/peel bond strength of orthodontic attachments to composite resins. Master thesis, University of Manitoba, Department of Preventive Dental Science Faculty of Dentistry, Canada, 2000.

12. Al-Dabbagh F. Evaluation of the effect of different mechanical surface roughness to improve repair bond

13. strength of aged composite restoration. Master thesis. Orthodontic department, University of Baghdad, 2008.

14. Gamra NMH, Kadhum AS, Yassir YA. An in vitro evaluation of shear bond strength of chemical and light cure bonding material with stainless steel, ceramic and sapphire brackets. J Bagh Coll Dentistry 2011; 23(2):133-8.

15. Bishara SE, Oonsombat C, Solimann MM, Warren JJ, Laffoon JF, Ajloni R. Comparison of bonding time and adhere bond strength between Conventional and a new integrated bonding system. Angle Orthod 2005; 75(2):23742.

16. Nemeth BR, Wiltshire WA, Lavaelle CLB. Shear/peel bond strength of orthodontic attachment to moist and dry enamel. Am j Orthod. Dentofac. Orthop 2006;129(3):396-401.

17. Uysal T, Sisman A. Can previously bleached teeth be bonded safely using Self-etching primer systems?. Angle Orthod 2008;78(4):711-5.

18. Bishara SE, Solimann MMA, Oonsombat C, Laffon JF, Ajlnoni R. The effect of variation in mesh-base design on the shear bond strength of orthodontic brackets. Angle Orthod 2004; 74(3): 400-4.

19. Polate Q, Karaman AL, Buyukyilmaz T. In vitro evaluation of shear bond strength and in vivo analysis of bond survival of indirect bonding regim. Angle Orthod 2004;74(3):405-9.

20. Wang WN, Meng CL, Tarng TH. Bond strength: A comparison between chemical coated and mechanical interlock bases of ceramic and metal brackets. Am J Orthod Dentofac Orthop 1997; 111(2):374-81.

21. Haselton DR, Diaz-Aronld AM, Vargas MA. Flexural strength of provisional crown and fixed partial denture resins. J Prosthet Dent 2002; 87(2):225-8.
22. Yap AU, Lye KW, Sau CW. Effect of aging on repair of resin modified glass- ionomer cement. J Oral Rehabil 2000; 27(5):422-7.

23. Rathke A, Tymina Y, Haller B. Effect of different surface treatments on the composite-composite repair bond strength. Clin Oral Investig 2009; 13(2):317-23.

24. Azarbal P, Boyer DB, Chan KC. The effect of the bonding agents on the interracial bond strength of repaired composites. Dent Mater J 1986; 2(2):153-5.

25. Li J. Effects of surface properties on bond strength between layers of newly cured dental composites. J Oral Rehab 1997; 24(5): 358-60.

26. Craig RG, Power JM. Restorative dental material, $11^{\text {th }}$ ed. Louis Mosby, 2002.

27. Scougall Vilchis RJ, Yamamoto S, Kitai N, Hotta M, Yamamoto K. Shear bond strength of a new fluoridereleasing orthodontic adhesive. Dent Mater J 2007; 26(2):45-51.

28. Velo S, Carano A, Carano A. Self-etching vs. traditional bonding systems in orthodontics: an in vitro study. Orthod Craniofac Res 2002; 5(3):166-9.

29. Fieker JP. Anew self-curing resin-modified glass-ionomer cement for direct bonding of orthodontic bracket in vivo. Am J Orthod Dentofac Orthop 1998; 113(4):384-6.

30. Brantly WA, Eliades T. Orthodontic material scientific and

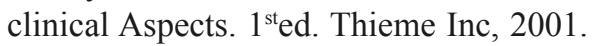

31. Harari D, Shapira-Davis S, Gillis I, Roman I, Redilich M. Tensile bond strength of ceramic brackets bonded to porcelain facets. Am j Orthod. Dentofac. Orthop 2003;123(5):551-4.

32. Sarac YS, Turk SE, Sarac D, Turk T. Surface condition methods and polishing technique effect on surface roughness of a feldspar ceramic. Angle Orthod 2007;77(4):723-8.

33. Al-Shamaa YA. Evaluation of the shear bond strength of metal bracket bonded to porcelain restoration (In vitro study). Master thesis. Orthodontic Department, University of Baghdad, 2009.

34. Ajloni R, Bishara SE, Oonsombat C, Solimand M, Laffoone J. The effect of the porcelain surface conditioning on bonding orthodontic brackets. Angle Orthod 2005; 75(5):858-64. 